International Journal of Engineering \& Technology, 7 (1.9) (2018) 141-144
International Journal of Engineering \& Technology
WPC
Website: www.sciencepubco.com/index.php/IJET
Research Paper

\title{
Computer aided drug design using virtual screening and molecular energy calculation of a specific neurodegenerative diseases
}

\author{
Bipin Nair B J ${ }^{1 *}$, Akshay Rajendran ${ }^{1}$ \\ ${ }^{1}$ Department of computer science Amrita School of Arts and Sciences, Mysuru Campus Amrita Vishwa Vidyapeetham, India \\ *Corresponding author E-mail: bipin.bj.nair@gmail.com
}

\begin{abstract}
Computer-aided drug design (CADD) is designing a drug with the help of computational algorithms. Information technology advances to creates the structure of molecules, molecular modeling and calculate the binding energies of the drug to initiate a new medicine against neurodegenerative diseases. In our work, we implemented virtual screening of a drug-protein interaction is selected from drug data bank with potential drug bank inhibitory activity for a specific neurodegenerative disease. Here we analyze technical CADD studies of the neurodegenerative diseases. Finally selecting the best alkaloid for a specific neurodegenerative disease and predicting the efficiency using computation of alkaloid with molecular energy.
\end{abstract}

Keywords: Computer Aided Drug Design; Neurodegenerative Diseases; Virtual Screening; Molecular Energy Calculation.

\section{Introduction}

Bioinformatics is a field of computation technique estimate an approach and advanced software tool for analyzing biological data. We are using biology and informatics, is evidently related to computational methods in to developing structure and identification of molecules. It is a mainly related between structure and compounds of biological data. Different computational implement and drug targets have made the drug release easy and definite because now only those cells can be targeted which are affected or change. It is also easy to know the molecular base of a disorder. Cheminformatic is used to the area of computation technique in chemistry. It combines to working in the field of chemistry, computational science, and information science. It is also applied to the data analysis for various kind of industries and medical fields. The application of cheminformatics is in the information related to compounds. It is used for structured data mining of database mining, graph mining, molecular mining, sequence mining etc. cheminformatics is represented in neurodegenerative diseases based on chemical structures, chemical markup language, SMILES. Here some formats are used to generate in 2 or 3 dimensions and learning physical interactions, modeling and docking studies.

Neurodegenerative disease is a neuron disease which effects in one generation to another. This disease if mostly affected on the aged people. It is an incurable condition which lost the neuron cell functions. Alzheimer's disorder (AD) is the greatest dementia in oldening population. Parkinson's disorder (PD), other nervous system disorders are related to the elderly peoples. The range of Alzheimer's, Parkinson's, of every neurodegenerative disorders as characteristic of major exploration efforts to determine crucial procedures in the development of neurodegenerative diseases.

\section{Literature survey}

Researchers are reported in the range of computational aided drugs to design in bioinformatics and comparing molecular energy calculation of specific neurodegenerative diseases as follows.

Bipin Nair BJ [1] was proposed the Inhibition are validated a therapeutic process for neurodegenerative diseases and exciting target for drugs representing studies using the Ligand and molecular structure-based methods. Faizul Azam [2] was introduced the chemical compound structure of the neurodegenerative disorder drug is an anesthetic medicine which incentives on treating the difficulties with aggression. Here developing the medicine using current drug developing methods that supports to vibrant the liabilities or the drug development methods produce the germs. Luciana Scotti [3] was proposed the work on computer-aided drug design to develop and interact with the medical discovery process associated with drug activity. Neurodegenerative diseases that used to be their drug property. CADD studies involve screening models for molecular separation, drug identification, biosynthetic metabolism, and drug interactions. Zeng, $\mathrm{H}$ [4] was introduced Alzheimer's disease (AD) is a disease characterized by the wildtype amyloid in the central nervous system. Alzheimer's disease is characterized as molecular structure-based drug screening (SBDS) or ligand-based drug screening (LBDS). Molecular dynamic is the first method here using etc. Morgan Robinson [5] was proposed by the Screening of the literature enabled us to find a limited number of AChE inhibitors that are suitable for QSAR analysis of acetylcholinesterase inhibitors for the treatment of Alzheimer's disease. Khurshid Ahmad [6] was suggested Parkinson's disease is characterized by tremor, stiffness and postural instability affected in central nervous system in neurodegenerative diseases. Here Lamarckian algorithm is used for molecular docking. Jhansi Rani Vangavaragu [7] was proposed the application of computer-based 
drug design is presented, collective with investigational actions, to discover the ability of various combinations to improve the hPreP proteolysis of this disesese. Cunha [8] was suggested Protein Aggregation in Neurodegenerative Diseases: Insights from Designs Using Computational Algorithms, Method, and Records Dedicated to Protein Aggregation and Amyloidosis. Richard.1. Jayaraj [9] was Studies were introduced in computer-aided drug design (CADD) to find potent inhibitors of alpha-synuclein aggregation in Parkinson's disease, a potent compound that binds to the active site of alpha-synuclein, can be professionally recognized. Anita Sarkar [10] was suggested Drugs and the system of drug delivery aiming amyloid- $\beta$ in the disease of Alzheimer's to use anti-A $\beta$ directing molecules effective of targeting $A \beta$, but the major difficulty to this method is the task posed by the barrier of blood-brain. Calvin Yu-Chian Chen [11] was suggested the machine of DJ1 function renovation by BAG1 through a method of computational simulation on Parkinson's disease-linked DJ1 transformation. $\mathrm{K}$.Y. Wong [12] was the prediction is focused on constraining the acetylcholinesterase (AChE) enzyme as a treatment of palliative in patients with an Alzheimer's disease. Anthony F. Nastase [13] was suggested Inhibition of BACE1 prevents the development or progression of amyloid- $\beta(A \beta)$ plaque formation and simple structure of Alzheimer's disease. P. K. Krishnan Namboori [14] was proposed Polymorphisms have been characterized using computational science and their complexity predicted by meditation of all mutations in the ApoE gene and their variation in individuals Here is the main application of tools, methods and calculation software analysis. Suresh Kumar A [15] was introduced the process is concerned with the analysis of mutations in the microsatellite regions of the above genes and to study the possibility of these mutations to cause Alzheimer's disease. Dorotea Rigamonti [16] was predicted to be introduced by small design molecules that can distress REST / NRSF nuclear translocation. The computational method was optimized in grouping with board and ligand-based drug design methods and functional consecutively to Huntington's disease. Elaine F. F. da Cunha. Speranta Avram [17] was proposed molecular technique simulation such as: 3D-QSAR (CoMFA, CoMSIA), rational drug design, and docking or variation of the molecular features will be detailed useful to Beta and Gamma secretase Inhibitors-Perspectives for an such kind of treatment of Alzheimer's diseases. Hong-Yu Zhang [18] was introduced In computer-aided drug design, there are fewer technical difficulties in finding more pluripotency drugs to implemented new strategies, namely, Alzheimer's disease in a single compound multitargeting strategy. Moussa B.H. Youdim [19] was suggested was predicted the neurodegenerative disorder treatments to use various CNS targets in multifunctional drugs. M. Recanatini [20] was predicted was proposed The work of docking acetylcholinesterase inhibitors against neurodegenerative diseases has been investigated by docking inhibition of the interaction between potential properties and $\mathrm{AChE}$ and molecular modeling techniques using piperidine molecular display studies

\section{Problem formulation}

These studies cover the range where we can combine Mycological, bio-chemoinformatic, pharmacologic, metabolic, biosynthetic, and molecular methods. It is very difficult getting an exact result from the medical field . so, the formation of a prototype and examine the all the possibilities in this area. When studying, drug design the many tasks included, molecular separation, identify the drugs, biosynthetic metabolism, and screening in molecular models will come across. Then docking Collaboration between two molecules within the drug as well as calculating the molecular energy for overcoming the constraint. These molecular structures are the interesting starting point for human inhibition studies for then we are using a cheminformatics tool for designing those structures. Species information as reported in the literature, but the compound isolation is not mentioned.

\section{Problem definition}

Here we are doing the computer-aided drug design using neurodegenerative diseases, data and inhibitors collected from the drug bank. Then neurodegenerative drugs are classified as well as remove the duplicate data which is selected from a common drug. Then predict the $3 \mathrm{~d}$ structure or designed drug with the combination of alkaloids. This will be docking with protein from the PDB for neurodegeneration proteins. Then calculate the energy variation of alkaloid with protein. Then performing a selected component analysis. From the $3 \mathrm{~d}$ generation describe drugs to generate descriptors from a trained group of drugs using random forest and cross-validation. the final testing of the particular model classified and classification of alkaloid as an active model. From our work, we are concentrating on a particular neurodegenerative disease and one particular screening method, then finally calculating the alkaloid molecular energy based on that selecting the compound. The selected compound is analyzed which is more efficient or not. The efficient compound is more useful for the neurodegenerative diseases.

\section{Architecture diagram}

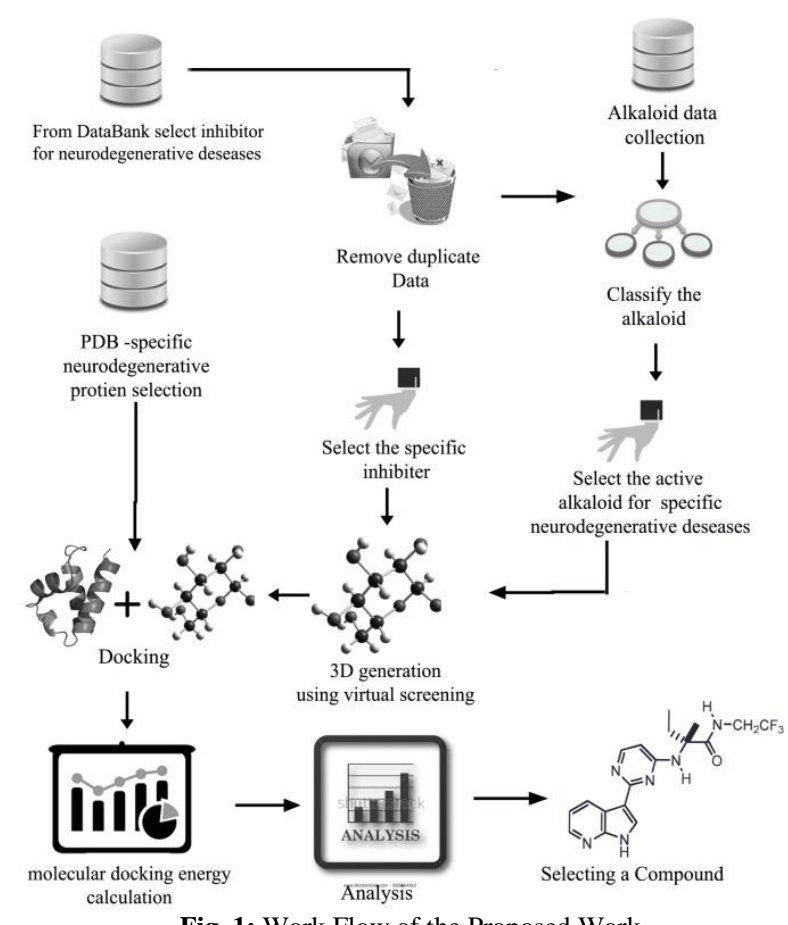

Fig. 1: Work Flow of the Proposed Work.

\section{Algorithm}

Classification algorithm for drug

Step 1: select the different type's neurodegenerative diseases.

Step 2: The collection of drug in the database search the type of neurodegenerative disease related to the drug.

Step 3: If the selected type of diseases equals to the drug's use, insert the drug into the classified-array.

Molecular structure generation algorithm

1) function DOCK(protein, ligand)

2) generate initial population poses at random

3) energies=COMPUT-ENERGIES(protein,drug, poses)

4) for each iteration of EMC do

5) select poses with lowest energies as parents

6) generate new population poses from parents

7) energies=COMPUTE-ENERGIES (protein, ligand, poses)

8) end for

9) output best poses

10) end function

11) function COMPUTE-ENERGIES(protein, ligand, poses) 
12) for $i=0$ up to size(poses) -1 do

13) transform ligand by poses [i]

14) energies $[\mathrm{i}]=0$

15) for each atom l-atom in ligand do

16) for each atom $p$-atom in protein do

17) energies[i]=energies[i]+INTERACTION(p-atom, i-atom)

18) end for

19) end for

20) end for

21) return energy

22) end function

\section{Dataset}

here we are using data set as set of neurodegenerative drugs.We are dedesigning a drug for treating the affected aged people. Here we using a collection of inhibitors from the drug bank. In the drug bank, we select an alternative drug of neurodegenerative diseases for depresion, rigidity, etc. Select an appropriate drug to use the interaction between the proteins.

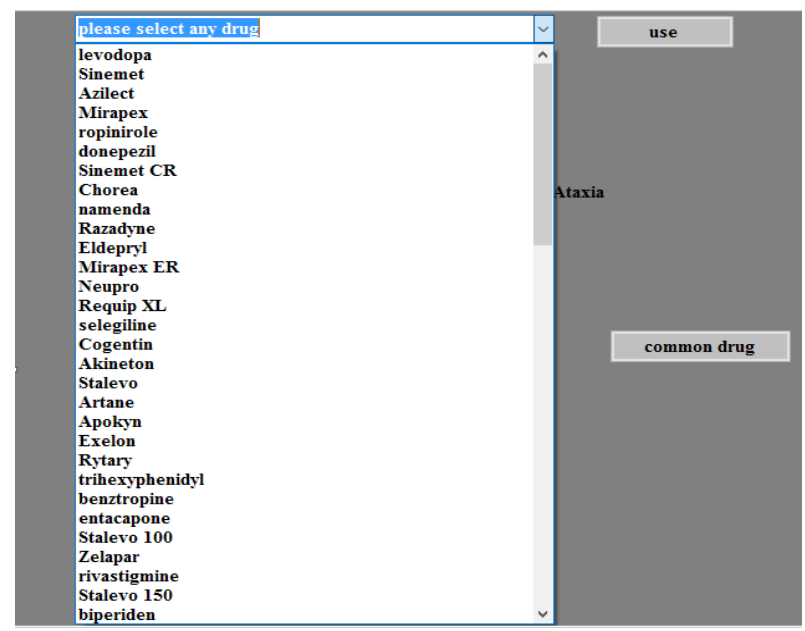

Fig. 2: Drug Collection.

Alkaloid collection- The collection of alkaloids related to the neurodegenerative disease. In that collection, we are selecting an alternative alkaloid to use for Alzheimer's or any other neurodegenerative diseases with the classification algorithm. The selected alkaloid is used to react with the neurodegenerative drug. The alkaloid is the substance of extracted natural source for the treatments of neurodegenerative diseases such as Parkinson's etc.

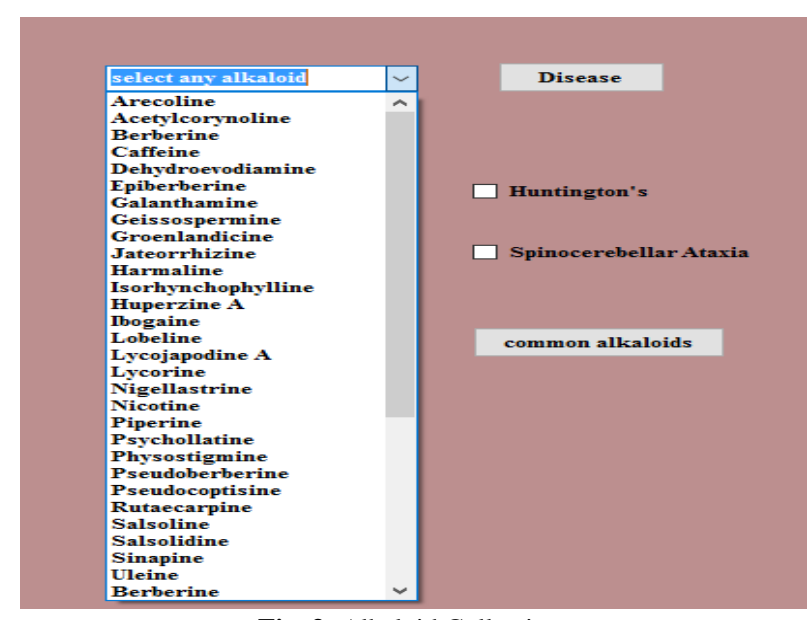

Fig. 3: Alkaloid Collection.

Protein collection- The protein collection also related to the neurodegenerative disease. From that we are collecting to classify and select an alternative protein from the PDB. In that protein is docking with the drug which is selected within the neurodegenerative disease classification. The protein is docking with the drug to calculate the molecular binding energy for checking the efficiency.

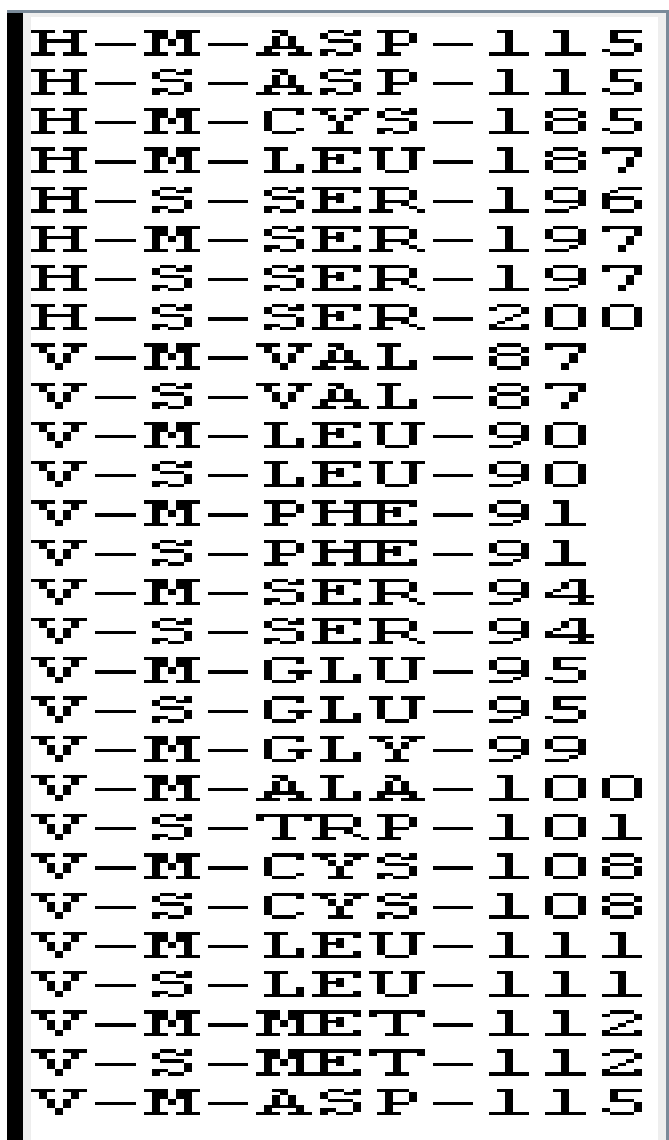

Fig. 4: Amino Acid of Receptors.

\section{Experimental result}

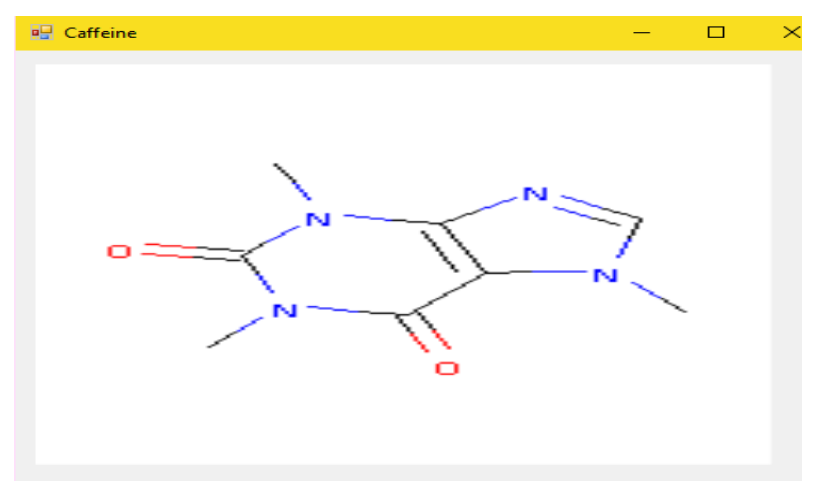

Fig. 5: 2D Structure of Caffeine Alkaloid.

From the classification of alkaloids, caffeine is the most popular drug and psychoactive substance in the world. Caffeine alkaloids are mainly used for Parkinson's disease. From the protection of neurons to the management of motor and non-motor symptoms. This disease is the most common second neurodegenerative disease, accounting for about $1 \%$ of the population over 60 years of age. Caffeine alkaloids are another agent that uses natural compounds. This means that it contains mainly nitrogen atoms. His pure caffeine is also known as trimethylxanthine and is highly water-soluble. In this alkaloid causes a level of dopamine increase which produces a good state of mind. 


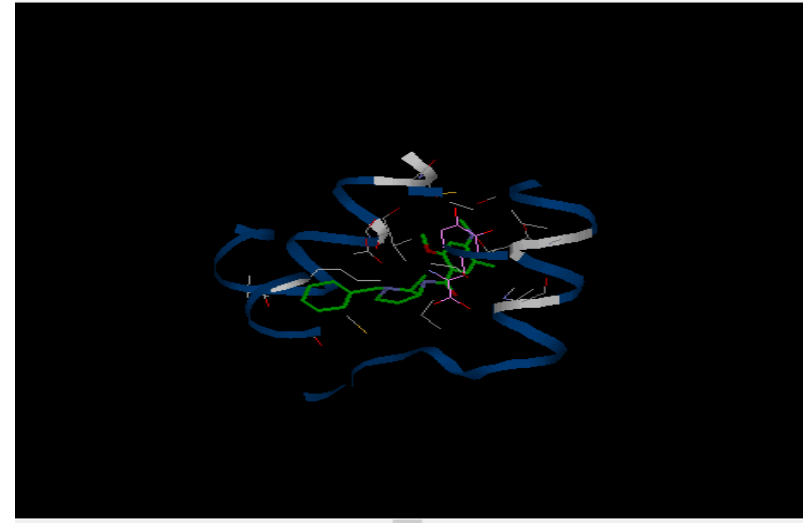

Fig. 6: 3d Structure Generation of Drug and Protein Docking.

Computational docking was developed for the generation of protein-drug interactions and efficient components for drug development. Here's the docking method for structure-based virtual filtering. Virtual screening is used to design drugs and other biological tools. And evaluate the accuracy of the docking method for improving the treatment.

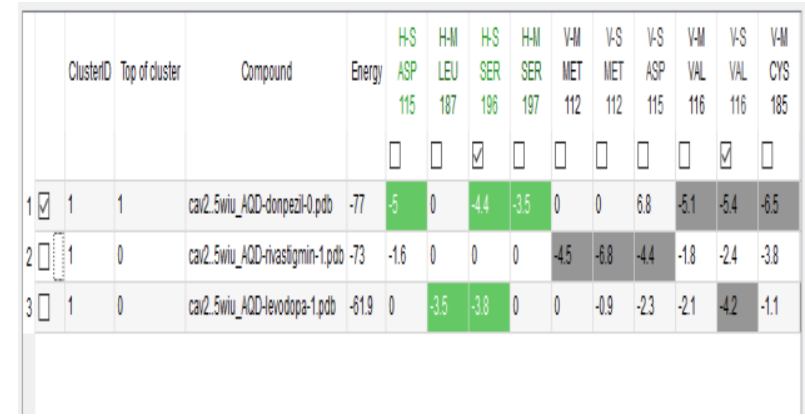

Fig. 7: Binding Energy Calculation.

Binding energy is the energy essential to take apart a complete system of sperate parts. It is calculated by the drug and protein interaction to analyze which is more efficient. Here the above fig7 shows the binding energy calculation of three drugs donepezil ($77)$, levodopa (-73), rivastigmine (-61.9). From the binding energy, we can check the analysis and select an active compound. This analysis express donepezil-protein interaction is very effective compound for the treatments neurodegenerative diseases.

\section{Conclusion}

In this paper, we analyze Computer-aided drug design involves predicting the efficiency of medications in the treatment of neurodegenerative diseases. Including neuronal diseases in the central nervous system, such as every neurogenerative diseases. From this paper we analyze a proper medicine for all these diseases. Levodopa drug is used to control the Alzheimer's. This drug has interacted with a neurodegenerative alkaloid from the alkaloid data collection. That compound is docking with protein. Recent studies, consuming the secondary metabolites with CADD. It analyzes with other drugs and selects a better compound for the neurodegenerative diseases. The analysis shows donepezil is the efficient compound of protein-drug docking.

\section{References}

[1] Nair, B. B., Bhaskaran, V., \& Arunjit, K. (2017). Structural designing of suppressors for autisms spectrum diseases using molecular dynamics sketch. International Journal of Drug Delivery, 8(4), 142-146. https://doi.org/10.5138/09750215.1973.

[2] Azam, F., Mohamed, N., \& Alhussen, F. (2015). Molecular interaction studies of green tea catechins as multitarget drug candidates for the treatment of Parkinson's disease: computational and structural insights. Network: Computation in Neural Systems, 26(3-4), 97-115.
[3] Scotti, L., \& Tullius Scotti, M. (2015). Computer aided drug design studies in the discovery of secondary metabolites targeted against agerelated neurodegenerative diseases. Current topics in medicinal chemistry, 15(21),

https://doi.org/10.2174/1568026615666150610143510.

[4] Zeng, H., \& Wu, X. (2016). Alzheimer's disease drug development based on Computer-Aided Drug Design. European journal of medicinal chemistry, 121, 851-863. https://doi.org/10.1016/j.ejmech.2015.08.039.

[5] Robinson, M., Lee, B. Y., \& Leonenko, Z. (2017). Drugs and Drug Delivery Systems Targeting Amyloid- $\mathrm{lb}$ \{eta $\}$ in Alzheimers Disease. arXiv preprint arXiv:1704.08313

[6] Ahmad, K., M Balaramnavar, V., H Baig, M., K Srivastava, A., Khan, S., \& A Kamal, M. (2014). Identification of potent caspase-3 inhibitors for treatment of multi-neurodegenerative diseases using pharmacophore modeling and docking approaches. CNS \& Neurological DisordersDrug Targets (Formerly Current Drug Targets-CNS \& Neurological Disorders), 13(8), https://doi.org/10.2174/1871527313666141023120843.

[7] Vangavaragu, J. R., Valasani, K. R., Gan, X., \& Yan, S. S. (2014). Identification of human presequence protease (hPreP) agonists for the treatment of Alzheimer's disease. European journal of medicinal chemistry, 76, 506-516. https://doi.org/10.1016/j.ejmech.2014.02.046.

[8] da Cunha, E. F., Resende, J. E., Franca, T. C., Gonçalves, M. A., de Souza, F. R., Santos-Garcia, L., \& Ramalho, T. C. (2013). Molecular modeling studies of piperidine derivatives as new acetylcholinesterase inhibitors against neurodegenerative diseases. Journal of Chemistry, 2013. https://doi.org/10.1155/2013/278742.

[9] Jayaraj, R. L., Ranjani, V., Manigandan, K., \& Elangovan, N. (2013). Insilico docking studies to identify potent inhibitors of alpha-synuclein aggregation in Parkinson Disease. Asian J Pharm Clin Res, 6(4), 127 131.

[10] Sarkar, A., Kumar, S., Grover, A., \& Sundar, D. (2012). Protein aggregation in neurodegenerative diseases: Insights from computational anal-

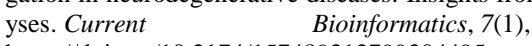
https://doi.org/10.2174/157489312799304495.

[11] Chen, C. Y. C. (2012). Mechanism of BAG1 repair on Parkinson's disease-linked DJ1 mutation. Journal of Biomolecular Structure and Dynamics, 30(1), 1-12. https://doi.org/10.1080/07391102.2012.674182.

[12] Y Wong, K., R Duchowicz, P., G Mercader, A., \& A Castro, E. (2012). QSAR applications during last decade on inhibitors of acetylcholinesterase in Alzheimer's disease. Mini reviews in medicinal chemistry, 12(10), 936-946. https://doi.org/10.2174/138955712802762365.

[13] Nastase, A. F., \& Boyd, D. B. (2012). Simple structure-based approach for predicting the activity of inhibitors of beta-secretase (BACE1) associated with Alzheimer's disease. Journal of chemical information and modeling, 52(12), 3302-3307. https://doi.org/10.1021/ci300331d.

[14] Namboori, P. K., Vineeth, K. V., Rohith, V., Hassan, I., Sekhar, L., Sekhar, A., \& Nidheesh, M. (2011). The ApoE gene of Alzheimer's disease (AD). Functional \& integrative genomics, 11(4), 519-522. https://doi.org/10.1007/s10142-011-0238-z.

[15] Kumar, S., Shilpa, S., Anil Kumar, N. C., Rohith, V., \& PK, K. N. (2010). Characterization of Microsatellite Regions of the Genes Causing 'Alzheimer's Disease'. Int. J. of Recent Trends in Engineering and Technology, 4(2).

[16] Rigamonti, D., Mutti, C., Zuccato, C., Cattaneo, E., \& Contini, A. (2009). Turning REST/NRSF dysfunction in Huntington's disease into a pharmaceutical target. Current pharmaceutical design, 15(34), 3958 3967. https://doi.org/10.2174/138161209789649303.

[17] Avram, S., Milac, A. L., Mihailescu, D. F., Dabu, A., \& Flonta, M. L. (2006). Computer-Aided Drug Design Applied to Beta and Gamma Secretase Inhibitors-Perspectives for New Alzheimer Disease Therapy. Current Enzyme Inhibition, 2(4), 311-328. https://doi.org/10.2174/157340806778699307.

[18] Zhang, H. Y. (2005). One-compound-multiple-targets strategy to combat Alzheimer's disease. FEBS letters, 579(24), $5260-5264$. https://doi.org/10.1016/j.febslet.2005.09.006.

[19] Youdim, M. B., \& Buccafusco, J. J. (2005). Multi-functional drugs for various CNS targets in the treatment of neurodegenerative disorders. Trends in Pharmacological Sciences, 26(1), 27-35. https://doi.org/10.1016/j.tips.2004.11.007.

[20] Recanatini, M., Cavalli, A., \& Hansch, C. (1997). A comparative QSAR analysis of acetylcholinesterase inhibitors currently studied for the treatment of Alzheimer's disease. Chemico-biological interactions, 105(3), 199-228. https://doi.org/10.1016/S0009-2797(97)00047-1. 